\title{
Consistencia interna y estructura factorial del Perfil de Impacto de Salud Oral aplicado a la enfermedad periodontal en una muestra de población general de adultos mexicanos
}

Internal Consistency and Factor Structure of the Oral Health Impact Profile Applied to the Periodontal Disease in a Mexican Adult General Sample

\author{
José Moral de la Rubia \\ Universidad Autónoma de Nuevo León, México \\ jose_moral@hotmail.com
}

Norma Idalia Rodríguez Franco

Universidad Autónoma de Nuevo León, México

perio2001@yahoo.com.mx
DOI: https://doi.org/10.11144/Javeriana.uo36-77.cief Redalyc: http://www.redalyc.org/articulo.oa? id $=231254499015$

Fecha de recepción: 31 Enero 2017 Fecha de publicación: 20 Septiembre 2017

\section{Resumen:}

Antecedentes: El Perfil de Impacto de Salud Oral (OHIP-14) es el instrumento más empleado para evaluar cómo influye la enfermedad bucal en la calidad de vida con respecto a la salud oral y la eficacia de tratamientos odontológicos. En México, el OHIP-14 se ha adaptado para aplicarse a la enfermedad periodontal (OHIP-14-PD). Se desconocen las propiedades métricas del OHIP-14-PD en población general y no está bien establecido el modelo factorial del OHIP-14. Objetivos: Describir la distribución, la discriminabilidad y la consistencia interna de los ítems y de la puntuación total del OHIP-14-PD; explorar su estructura factorial, y contrastar varios modelos factores, estudiando la consistencia interna, validez convergente y discriminante de los factores. Métodos: Se aplicó el OHIP-14-PD a una muestra de 256 adultos de población general de Monterrey, México. Se usó análisis factorial confirmatorio. Resultados: La consistencia interna del OHIP-14-PD fue muy alta $(\alpha$ ordinal $=0,924)$. Su distribución mostró asimetría y curtosis positivas con una media de 12,32 (intervalo de confianza del 95 \%: [11,29, 13,34]) y una mediana de 10,5. El modelo de un factor se sustentó por análisis paralelo de Horn y tuvo un ajuste de bueno (GFI = 0,976, AGFI $=0,967, \mathrm{NFI}=0,968$ y RFI $=0,962)$ a adecuado $(\chi 2 / \mathrm{gl}=2,336$ y SRMR $=0,083)$. Al contemplar la relación entre dos pares de residuos, el ajuste resultó bueno por todos los índices y el factor tuvo validez convergente. Conclusión: El OHIP-14-PD constituye una medida unidimensional con consistencia interna y validez convergente.

Palabras clave: adultos, análisis factorial, enfermedad periodontal, estudios de validación como tópico, instrumentos de evaluación, población general, salud oral.

Áreas temáticas: calidad de vida en relación con la salud oral; evaluación; odontología; periodoncia

\begin{abstract}
:
Background: The Oral Health Impact Profile (OHIP-14) is the most frequently used instrument to evaluate how an oral disease impacts on the patient's life quality based on his/her oral health and the dental treatment efficacy. In Mexico, the OHIP-14 has been adapted to be applied in the periodontal disease cases (OHIP-14-PD). The measuring properties of the OHIP-14-PD are unknown for the general population and are not duly established for the OHIP-14 factor model. Objectives: To describe the distribution, breakdown and internal consistency of the items and total score in the OHIP-14-PD; to explore the factor structure and to compare different factor models, thus studying the internal consistency, both converging and discriminating validity of the factors. Methods: The OHIP-14-PD was applied in a sample consisting of 256 common adults from Monterrey, Mexico. At the end, a factor analysis was used for confirmation. Results: The OHIP-14-PD internal consistency was high (ordinal $\alpha=0.924)$. the distribution showed asymmetries and positive kurtosis with a mean value of 12.32 (95\% CI: [11.29, 13.34]) and a median value of 10.5. The factor model was supported by the Horn parallel analysis and showed an adjustment from $\operatorname{good}(\mathrm{GFI}=0.976, \mathrm{AGFI}=0.967, \mathrm{NFI}=0.968$ and $\mathrm{RFI}=0.962)$ to appropriate $(\chi 2 / \mathrm{gl}=2.336$ and $\mathrm{SRMR}=0.083)$. When observing the relationship between two residual pairs, the adjustment resulted to be good for all the indexes and the factor showed a converging validity. Conclusion: The OHIP-14-PD is a one-dimension parameter with internal consistency and converging validity.
\end{abstract}

Keywords: adults, factor analysis, periodontal disease, topic validation studies, evaluation instruments, general population, oral health.

Thematic fields: assessment; periodontics; quality of life in relation to oral health 


\section{INTRODUCCIÓN}

Examinar las propiedades métricas de un instrumento de autorreporte, aplicable en la clínica e investigación odontológicas, es una tarea importante y poco abordada dentro de esta disciplina. Este artículo empírico se centra en los aspectos métricos de una escala que evalúa el impacto de la enfermedad periodontal sobre la calidad de vida relacionada con la salud bucal. Estas propiedades se estudian en adultos mexicanos de la población general. En relación con la enfermedad periodontal en México, cabe señalar que su prevalencia aumenta a mayor edad. Solo el $40 \%$ de los pacientes adultos de 35 a 44 años presentan un periodonto sano. Este porcentaje disminuye al $30 \%$ en adultos mayores de 65 a 74 años. Nacionalmente, la enfermedad periodontal es la segunda causa de morbilidad bucodental, precedida por la caries dental, por lo que representa un problema de salud pública que afecta a la población (1).

Un instrumento de medida usado en la investigación y la clínica odontológica es el Perfil de Impacto sobre la Salud Oral (OHIP) (2,3). Esta escala de autorreporte evalúa la frecuencia con que se experimentan dificultades para realizar ciertas funciones y actividades de la vida diaria, debido al padecimiento de un trastorno bucal (2). Se basa en el modelo teórico multidimensional de salud propuesto por la Organización Mundial de la Salud (4). Locker (5) adaptó este modelo a la salud oral, y Slade y Spencer (2) crearon el OHIP-49 con base en dicha adaptación. Inicialmente, el instrumento estuvo conformado por 49 ítems con 5 categorías ordenadas de respuesta y un modelo de 7 factores: limitación funcional, dolor físico, discapacidad física, malestar psicológico, discapacidad psicológica, discapacidad social y minusvalía. Posteriormente, Slade (3) validó el modelo de 7 factores y sugirió simplificar el instrumento a los 2 indicadores con más peso en cada factor, con lo que definió el OHIP-14. La estructura factorial del OHIP-14 se ha estudiado bajo 3 enfoques: factorial exploratorio (6), factorial confirmatorio $(7,8)$ y con ambos métodos $(9,10)$.

En pacientes odontológicos australianos, desde el enfoque factorial exploratorio, Brennan y Spencer (6) encontraron dos factores: limitación funcional-incapacidad física (ítems 1, 2, 5, 6, 7, 8, 9, 10, 12 y 13) y aspectos psicosociales de la discapacidad y minusvalía (ítems 3, 4, 11 y 14), de los cuales los ítems 4, 8 y 12 tenían saturaciones altas en ambos factores.

Desde el enfoque factorial confirmatorio, en una muestra de adolescentes brasileños, Silveira y colaboradores (7) contrastaron un modelo jerarquizado de un factor de orden superior (severidad de impacto) y 7 factores de orden inferior con 2 indicadores cada uno desde la propuesta original de Slade (3). El ajuste del modelo fue aceptable por máxima verosimilitud: $\chi^{2} / \mathrm{gl}=3,973$; $\mathrm{CFI}=0,957$; GFI = 0,959; y $\mathrm{RMSEA}=$ 0,063 . En pacientes adultos brasileños atendidos en una clínica dental universitaria, Zucoloto y colaboradores (8) estimaron la bondad de ajuste (análisis unigrupo) y la invarianza (análisis multigrupo) de 2 modelos jerarquizados, usando el método de máxima verosimilitud. Hallaron que el modelo con mejores propiedades fue el de 3 factores de orden superior correlacionados (impacto sobre la salud oral: físico, psicosocial y social) y 7 factores de orden inferior. Los factores de limitación funcional, dolor físico y discapacidad física se jerarquizaron al factor de impacto físico; los factores de malestar psicológico y discapacidad psicosocial, al factor de impacto psicosocial, y los factores de discapacidad social y minusvalía, al factor de impacto social. En la muestra total, las cargas factoriales de los 7 factores jerarquizados variaron de 0,54 a 0,84 ; la varianza media extraída, de 0,46 a 0,63; la varianza compartida entre los 7 factores, de 0,21 a 0,50; y la consistencia interna por la alfa de Cronbach, de 0,62 a 0,77, y por el coeficiente de fiabilidad compuesta, de 0,63 a 0,77. Se juzgó el ajuste como aceptable $\left(\chi^{2} / \mathrm{gl}=5,07\right.$; GFI = 0,97; AGFI = 0,96; RMSEA =0,06).

Desde un enfoque factorial mixto, en trabajadores españoles, Montero y colaboradores (9) hallaron 3 factores en el análisis exploratorio: impacto psicosocial (ítems 5, 6, 9, 10, 11, 12, 13 y 14), dolor/malestar (ítems 3, 4, 7 y 8) y limitación funcional (ítems 1 y 2). Este modelo de 3 factores correlacionados tuvo un ajuste aceptable por máxima verosimilitud $\left(\chi^{2} / \mathrm{gl}=2,09\right.$; CFI= 0,95; RMSEA $\left.=0,06\right)$. Mello dos Santos y colaboradores (10) proponían un modelo unifactorial con buen ajuste en una muestra de mujeres brasileñas 
postparto (RMSEA $=0,04 ; \mathrm{CFI}=0,98 ; \mathrm{TLI}=0,98)$ y en una muestra de adultos mayores de ambos sexos ( $\mathrm{RMSEA}=0,04 ; \mathrm{CFI}=0,98 ; \mathrm{TLI}=0,98$ ) usando máxima verosimilitud.

Actualmente, el OHIP-14 es el instrumento más empleado para evaluar cómo repercute la enfermedad bucal en la calidad de vida respecto a la salud bucal y la eficacia de tratamientos odontológicos (11). En México, Rodríguez y Moral (12) adaptaron el OHIP-14 para evaluar el efecto de la enfermedad periodontal sobre la calidad de vida en relación con la salud oral, generando el OHIP-14-PD. En dicho estudio (12) se estableció la validez de contenido del OHIP-14-PD usando un juicio de expertos. En otro estudio de validación del OHIP-14-PD, realizado en una muestra clínica de 249 pacientes adultos con periodontitis y gingivitis, se concluyó que el modelo unifactorial era el más adecuado. El modelo de un factor mostró un ajuste de bueno (GFI $=0,976$; $\mathrm{AGFI}=0,967 ; \mathrm{NFI}=0,968 ; \mathrm{RFI}=0,962)$ a adecuado $\left(\chi^{2} / \mathrm{gl}=2,336\right.$ y SRMR $=0,083)$ por mínimos cuadrados libres de escala. La consistencia interna fue muy alta $(\alpha$ ordinal $=0,928), y$ la distribución de la puntuación normal mostró asimetría positiva y apuntamiento con una media de 18,01 (intervalo de confianza [IC] del $95 \%:[16,62,19,40]$ ) y mediana de $17(13)$.

Con respecto a las propiedades métricas del OHIP-14-PD, hay 2 vacíos de conocimiento. Por una parte, todavía no existe un modelo factorial bien establecido para sus 14 ítems, considerando la diversidad de propuestas con su precursor, que es el OHIP-14. Por otra, aunque las propiedades del OHIP-14-PD se han estudiado en población clínica odontológica, estas se desconocen en la población general. Considerando estos dos vacíos de conocimiento, en la presente investigación se plantearon como objetivos: 1) describir la distribución, comprobar la discriminabilidad y calcular consistencia interna de los 14 ítems y de la puntuación total del OHIP-14 en una muestra de adultos de población general de la ciudad de Monterrey en México; 2) explorar la estructura factorial de sus 14 ítems; 3) contrastar los modelos factores propuestos por Moral y Rodríguez (13), Silveira y colaboradores (7), Zucoloto y colaboradores (8), Montero y colaboradores (9), Mello dos Santos y colaboradores (10) y los derivados del análisis factorial exploratorio (AFE); y 4) estudiar la consistencia interna y la validez convergente y discriminante de los factores en cada modelo factorial contrastado. Debe matizarse que, en la presente investigación, se emplea el término adulto en un sentido amplio, indicando personas mayores de edad, y por tanto abarcando a postadolescentes (18 y 19 años) dentro del concepto.

\section{MATERIALES Y MÉTODOS}

Se realizó un estudio descriptivo correlacional con un diseño transversal. Se recolectó una muestra no probabilística incidental de población general adulta integrada por 256 participantes. Los criterios de inclusión fueron dar el consentimiento informado, edad de 18 años o mayor, saber leer y escribir, así como residir en Monterrey o en su área metropolitana. El criterio de exclusión fue presentar problemas clínicos de atención y comprensión, como esquizofrenia, autismo o demencia. El criterio de eliminación fue dejar, al menos, un dato incompleto en el OHIP-14-PD. Cabe señalar que no se hizo ningún examen periodontal en relación con algún criterio de inclusión o exclusión, por lo que la presente muestra no es una muestra control o de participantes sanos, sino de una muestra de población general de personas mayores de edad.

Los datos se recabaron durante el periodo octubre de 2015-marzo de 2016. Por un parte, las personas laboralmente activas se encuestaron en sus lugares de trabajo (oficinas, fábricas y restaurantes). Por otra parte, amas de casa, estudiantes, desempleados y personas jubiladas se encuestaron en sus domicilios particulares. Los lugares se eligieron por conveniencia o proximidad a la red de 5 colaboradores de investigación y los 2 responsables principales de la investigación, quienes recolectaron los datos.

Se invitó a los participantes a formar parte del estudio, explicándoles en qué consistía la investigación y se procedió a solicitar la firma del consentimiento informado. Se respetó la confidencialidad de la información 
en el manejo de los datos. El estudio recibió aprobación por el Comité de Bioética de la Secretaría de Salud (DEISC-19-01-16-16).

Para motivar la participación se obsequiaron productos de higiene bucal. Se logró un porcentaje de participación del $100 \%$. Además, se insistió en que no se dejasen preguntas sin responder y no se requirió eliminar ningún caso por valores perdidos en el OHIP-14-PD.

El cuestionario aplicado de autorreporte empezaba con la solicitud de firma del consentimiento informado. Seguían unas preguntas cerradas sobre información sociodemográficas (edad, sexo, municipio, estado civil, ocupación, escolaridad y estrato socioeconómico). Terminaba con la escala de Perfil de Impacto de Salud Oral aplicado a la enfermedad periodontal (OHIP-14-PD) (12). Esta escala se adaptó a partir del OHIP-14 de Slade (3), enfocando las preguntas en la enfermedad periodontal (figura 1). El OHIP-14 consta de 14 ítems distribuidos en 7 factores: limitación funcional (ítems 1 y 2), dolor físico (ítems 3 y 4 ), malestar psicológico (ítems 5 y 6), discapacidad física (ítems 7 y 8), discapacidad psicológica (ítems 9 y 10), discapacidad social (ítems 11 y 12) y minusvalía (ítems 13 y 14) (4). Las preguntas se responden bajo un formato cerrado de 5 categorías ordenadas: $0=$ nunca, $1=$ casi nunca, $2=$ ocasionalmente, $3=$ frecuentemente y $4=$ muy frecuentemente. La puntuación total del OHIP-14-PD se obtiene por la suma directa de los 14 ítems y tiene un rango de 0 a $56(3,12)$.

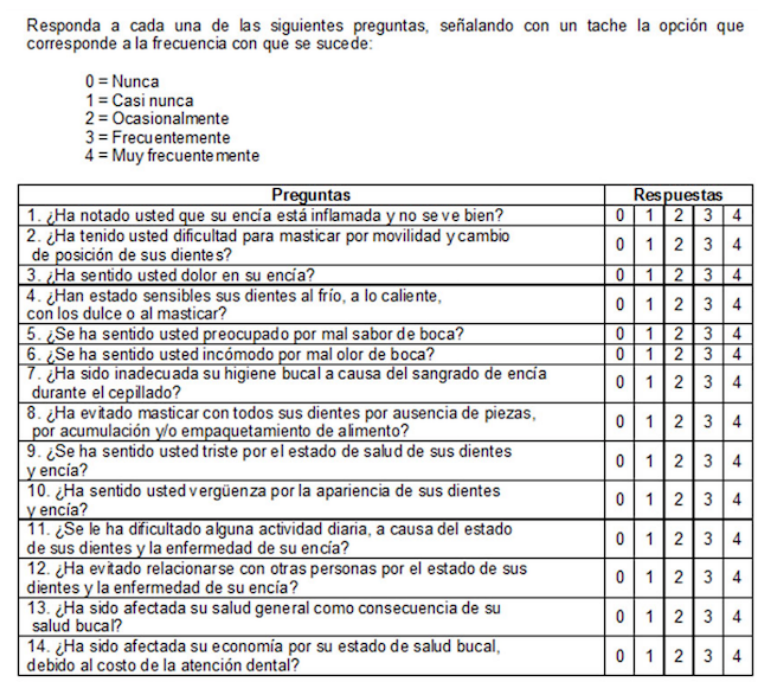

FIGURA 1

OHIP-14-PD. Perfil de Impacto sobre la Salud Oral aplicado a la enfermedad periodontal

Con respecto al análisis de datos, en primer lugar, se determinaron las propiedades de discriminabilidad y consistencia de los ítems. La discriminabilidad se estableció por la diferencia de tendencia central en el ítem entre el grupo de puntuaciones altas y bajas en la puntuación total del OHIP-14-PD. Los grupos se definieron por el primer cuartil y el tercero. La diferencia de tendencia central se contrastó por la prueba U de MannWhitney. La consistencia interna se estimó por la correlación del ítem con la suma de los restantes ítems y el efecto de la eliminación del ítem sobre la consistencia interna de la escala. La correlación se calculó por el coeficiente de correlación poliserial (CPS), y la consistencia interna, por el coeficiente alfa ordinal ( $\alpha$ ordinal $\mathrm{t}-\mathrm{i})$. Un ítem tiene consistencia débil en caso de que CPS $(\mathrm{i}, \mathrm{t}-\mathrm{i})<0,50$, e incrementa la consistencia interna

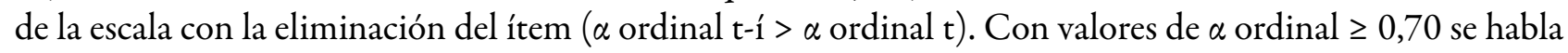
de consistencia interna aceptable; $\geq 0,80$ es alta $y \geq 0,90$ es unitaria (14). Además, se contrastó el ajuste a una distribución normal de la puntuación total en el OHIP-14-PD por la prueba de Kolmogorov-SmirnovLilliefors.

En segundo lugar, se determinó la estructura factorial de la escala usando AFE y confirmatorio. En el AFE, la extracción de factores se realizó por el método de mínimos cuadrados ponderados. Se rotó la matriz factorial por el método oblicuo Promax. Cada ítem se asignó al factor en el que presentase su carga más alta y mayor que 0,40 dentro de la matriz estructural (15). Para determinar el número de factores se contemplaron 6 criterios, calculados desde la matriz de correlaciones policóricas. Se buscó la convergencia de los criterios 
valorándolos en el siguiente orden de importancia: análisis paralelo de Horn (percentil 95), coordenadas óptimas, media mínima de las correlaciones parciales al cuadrado o a la cuarta potencia (criterio de Velicer), factor de aceleración y autovalores mayores que uno o criterio de Kaiser (16).

En el análisis factorial confirmatorio (AFC) se minimizó la función de discrepancia por el método de mínimos cuadrados libres de escala. Los errores estándar de los parámetros se estimaron y su significación se contrastó por el método de percentiles corregidos de sesgo, usando remuestreo paramétrico con la extracción de 2000 muestras. Se optó por el remuestreo paramétrico para generar los datos (matrices de correlaciones) bajo un modelo de distribución normal, al usarse la matriz de correlaciones policóricas como datos de entrada (17). Se usó un intervalo de confianza del $95 \%$ y un nivel de significación bilateral de 0,05. Los valores de correlación (coeficiente de correlación policórica entre los ítems y coeficiente de correlación productomomento de Pearson entre los factores $[r]$ ) y pesos de medida estandarizados $(\lambda)$ estuvieron entre $-0,299$ y $-0,10$ o entre 0,10 y 0,299 , y se interpretaron como fuerza de la asociación o tamaño del efecto pequeño; entre $-0,490$ y $-0,30$ o 0,30 y 0,499 , como medio; entre $-0,699$ y $-0,50$ o entre 0,50 y 0,699 , como grande; entre $-0,899$ y $-0,70$ o entre 0,70 y 0,899 , como muy grande, y entre -1 y $-0,90$ o 0,90 y 1 , como unitario (18).

Se contemplaron 6 índices de ajuste: chi-cuadrado relativo $\left(\chi^{2} / \mathrm{gl}\right)$, residuo estandarizado cuadrático medio (SRMS), índice de bondad de ajuste (GFI) de Jöreskog y Sörbom y su modalidad corregida (AGFI), índice normado de ajuste (NFI) de Bentler y Bonett e índice relativo de ajuste (RFI) por el coeficiente rho de Bollen. Se estipularon como valores de buen ajuste: $\chi^{2} / \mathrm{gl} \leq 2$; SRMS $\leq 0,08$; GFI $\geq 0,95$; AGFI $\geq 0,90$; NFI $\geq 0,95$; RFI $\geq 0,95$. Como valores de ajuste adecuado: $\chi^{2} /$ gl $\leq 3$; SRMS $<0,10$; GFI $\geq 0,90$; AGFI $\geq 0,85$; NFI $\geq 0,90 ; \mathrm{RFI} \geq 0,90$ (17). Se consideró que la bondad de ajuste entre dos modelos es equivalente cuando el cociente entre la diferencia de sus estadísticos chi y la diferencia de sus grados de libertad es menor o igual que $5\left(\Delta \chi^{2} / \Delta \mathrm{gl} \leq 5\right)$ y las diferencias en los índices GFI, NFI y RFI son menores o iguales que 0,01 (16). Se estimó la parsimonia del modelo por el índice (PR) de James, Mulaik y Brett. Se interpretaron valores de PR $\geq 0,75$ como parsimonia alta, $\geq 0,50$ media, $\geq 0,25$ baja $y<0,25$ muy baja (19).

Por último, se calculó la consistencia interna de los factores y se comprobó su validez convergente y discriminante. La consistencia interna se estimó por el coeficiente alfa ordinal. Para establecer la validez convergente de cada factor, se calculó la varianza media extraída (VME) por la media aritmética de los cuadrados de las cargas de sus ítems en la matriz estructural, así como la confiabilidad compuesta (CC) por el coeficiente omega de McDonald ( $\omega)$, usando estas mismas cargas. La combinación de una VME $>0,50$ y una $\mathrm{CC} \geq 0,70$ o una VME $>0,45$ y CC $\geq 0,80$ se tomó como criterio de validez convergente (14). Para establecer la validez discriminante se contemplaron dos criterios: 1) siguiendo el criterio de Fornell-Larcker, se consideró que un factor presenta validez discriminante con respecto a otro factor cuando el cuadrado de la correlación entre dos factores (varianza compartida) es mayor que la VME del factor y menor que dos tercios $\left.\left(\mathrm{VME}<\mathrm{r}^{2}<0,66\right)(20) .2\right)$ Usando el criterio del cociente de las correlaciones heterorrasgo-monorrasgo (CHRMR), se consideró que cuando el cociente entre la media aritmética de las correlaciones cruzadas entre los ítems de los dos factores y la media geométrica de las dos medias aritméticas de las correlaciones de los ítems de cada factor es menor que 0,85 hay validez discriminante (21). Los análisis se hicieron con SPSS22, menú R2,4 para SPSS22, AMOS16 y Excel 2007.

\section{RESULTADOS}

\section{Descripción de la muestra}

El 51,6 \% de los participantes correspondió a mujeres, y el 48,4\%, a hombres. La edad varió de 18 a 77 años con una media de 40,67. La escolaridad varió de primaria (6 años) a posgrado (al menos 18 años) con una 
mediana de estudios medios superiores técnicos (12 años). De los 256 participantes, el 30,5\% respondió ser solteros; el 57,4\%, ser casados; el 5,1 \%, ser divorciados; el 3,1 \%, ser viudos; el 1,2 \%, ser separados, y el 2,7 \%, vivir en unión libre. Con respecto a la ocupación laboral, el 12,1 \% dijo ser amas de casa; el $7 \%$, ser obreros; el 61,7\%, ser empleados; el 5,1\%, tener negocio propio; el 5,5\%, ser estudiantes; el 0,8 \%, estar desempleados, y el 7,8 \%, estar jubilados. En cuanto al estrato socioeconómico autodefinido (subjetivo), el 4,7\% se consideró de estrato bajo; el 26,6 \%, de medio-bajo; el 60,9\%, medio-medio; el 7,8 \%, medio-alto, y ninguno alto, con una mediana de estrato socioeconómico medio-medio.

\section{Distribución, discriminabilidad y consistencia interna de los ítems y puntuación total}

La mediana fue 1 en siete ítems, 0 en seis y 2 en uno. En ocho ítems hubo asimetría positiva ( $\left.A_{\text {IQ }}=1\right)$ y en uno asimetría negativa $\left(\mathrm{A}_{\mathrm{IQ}}=-1\right)$. Las distribuciones de los cinco ítems restantes fueron simétricas $\left(\mathrm{A}_{\mathrm{IQ}}=\right.$ $0)$, pero presentaron apuntamiento $\left(C_{p c}=0,327\right)$. Por las pruebas de Mardia, la distribución multivariada de los 14 ítems no se ajustó a una distribución normal (asimetría multivariada: $\chi^{2}=412,236, \mathrm{p}<0,001$; curtosis multivariada: $\left.\chi^{2}=1910,174, \mathrm{p}<0,001\right)$. Los 14 ítems mostraron discriminabilidad $(Z U<-6, \mathrm{p}<0,001)$. La consistencia interna fue alta en los 14 ítems $\left(\mathrm{r}_{\mathrm{ps}(\mathrm{t}-\mathrm{i})}>0,50 \mathrm{y} \alpha\right.$ ordinal $(\mathrm{t}-\mathrm{i})>\alpha$ ordinalt $\left.=0,928\right)$, salvo en los ítems 4 y 14 que presentaron correlaciones corregidas ligeramente menores que $0,50(\mathrm{rps}(\mathrm{t}-\mathrm{i})=0,488 \mathrm{y}$ 0,499 , respectivamente), pero su eliminación hizo disminuir el valor del coeficiente alfa ordinal (tabla 1 ).

TABLA 1

Distribución, discriminabilidad y consistencia interna de los ítems del OHIP-14-PD $\mathrm{n}=256$. Categorías ordenadas de respuesta: $\mathrm{N}=$ "Nunca" $=0, \mathrm{CN}=$ "Casi nunca" $=1, \mathrm{O}=$ "Ocasionalmente" $=2, \mathrm{~F}=$ "Frecuentemente" = 3, MF = "Muy frecuentemente" = 4. \% = porcentajes. AIQ = coeficiente de asimetría intercuartílica de YouleBowley. $\mathrm{Cpc}=$ curtosis percentílica corregida. Discriminabilidad: $\mathrm{Z}_{\mathrm{U}}=$ valor estandarizado del estadístico $\mathrm{U}$ de Mann-Whitney, ${ }^{* * *} \mathrm{p}<0,001$ (bilateral). CPS = correlación poliserial del ítem con la suma de los 14 ítems, $\mathrm{r}$ ps $(\mathrm{t}-\mathrm{i})=$ correlación poliserial del ítem con la suma de los restantes 13 ítems, $\alpha \mathrm{t}-\mathrm{i}=$ coeficiente alfa ordinal excluido el ítem ( $\alpha$ ordinal $=0,924$ para los 14 ítems).

\begin{tabular}{|c|c|c|c|c|c|c|c|c|c|c|c|c|}
\hline \multirow{2}{*}{ Îtem } & \multicolumn{5}{|c|}{ Distribución (\%) } & \multirow{2}{*}{ Mdn } & \multirow{2}{*}{$\mathbf{A}_{\mathrm{IQ}}$} & \multirow{2}{*}{$\mathrm{C}_{\mathrm{PS}}$} & \multirow{2}{*}{$\begin{array}{l}\text { Discrimi- } \\
\text { nabilidad }\end{array}$} & \multicolumn{3}{|c|}{ Consist. Interna } \\
\hline & $\mathbf{n}$ & $\mathrm{CN}$ & $\mathbf{O}$ & $\mathbf{F}$ & MF & & & & & $\mathbf{r}_{\mathrm{PS}}$ & $\mathbf{r}_{\mathrm{ns}(\mathrm{t}-\mathrm{i})}$ & $\boldsymbol{\alpha}_{t-i}$ \\
\hline 1 & 38,7 & 23,0 & 32,4 & 3.5 & & 1 & 0 & 0,237 & $-7,706^{* *}$ & & & 0,920 \\
\hline 1 & 5 & & & 2,0 & & 1 & 1 & & & & & 16 \\
\hline 3 & 41,4 & 30,5 & 24,2 & 3,1 & 0 & 1 & 0 & & -7 & 630 & & 0,919 \\
\hline 4 & 1 & 26,2 & 44,5 & 9,4 & 2,7 & 2 & -1 & 0 , & $-6,6$ & 847 & & 0,923 \\
\hline 5 & 22,3 & 32,0 & 35,2 & 8,2 & 2 & 1 & 1 & 0, & -7 & 0,645 & 23 & 0,920 \\
\hline 6 & 21,9 & 35,2 & 34,8 & 5,1 & 3,1 & 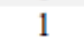 & 1 & 0,2 & -7 & 0,645 & 26 & 0,920 \\
\hline 7 & 40,2 & 29,7 & 24,6 & 3,5 & 2 & 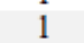 & 0 & 0,2 & -6 , & 0,564 & 0,516 & 0,922 \\
\hline 8 & 53,5 & 18,0 & 20,7 & 5,5 & 2,3 & 0 & 1 & & -8 & 0,630 & 0,582 & 0,920 \\
\hline 9 & 46,1 & 26,6 & 20,3 & 4,3 & 2,7 & 1 & 0 & 0,237 & $-9,580^{* *}$ & 0,813 & 0,810 & 0,913 \\
\hline 10 & 46,9 & 23,4 & 21,1 & 5,1 & 3,5 & 1 & 0 & 0,237 & $-8,869^{\cdots}$ & 0,759 & 0,741 & 0,915 \\
\hline 11 & 72,3 & 19,1 & 7,8 & 0,8 & 0 & 0 & 1 & 0,237 & $-8,742^{\ldots *}$ & 0,656 & 0,649 & 0,915 \\
\hline 12 & 81,6 & 12,1 & 4,7 & 1,2 & 0,4 & 0 & I & $-0,263$ & $-7,74$ & 0,585 & 0,571 & 0,916 \\
\hline 13 & 83,6 & 12,1 & 3,5 & 0,4 & 0,4 & 0 & I & $-0,263$ & $-7,495^{* *}$ & 0,587 & 0,577 & 0,916 \\
\hline 14 & 53,1 & 20,7 & 19,5 & 4,3 & 2,3 & 0 & 1 & 0,237 & $-7,682^{* *}$ & 0,561 & 0,499 & 0,921 \\
\hline
\end{tabular}

La consistencia interna de los 14 ítems fue muy alta $(\alpha$ ordinal $=0,924)$. La distribución de la puntuación total del OHIP-14-PD mostró asimetría positiva $\left(A_{F}=1,115\right.$, IC del 95 \%: $\left.[0,816,1,414]\right)$ y apuntamiento $\left(\mathrm{C}_{\mathrm{F}}=1,339\right.$, IC del $95 \%$ : $\left.[0,742,1,936]\right)$ por los coeficientes de Fisher, por lo que no se ajustó a una distribución normal $\left(\mathrm{Z}_{\mathrm{K}-\mathrm{S}-\mathrm{L}}=0,109, \mathrm{p}<0,001\right)$. La media fue 12,32 (IC del 95 \%: [11,29, $13,34])$ con una desviación estándar de 8,348 y la mediana fue $10,5\left(\mathrm{Q}_{1}=6\right.$ y $\left.\mathrm{Q}_{3}=17\right)$. 


\section{Análisis factorial exploratorio}

El número de factores fue uno por el análisis paralelo de Horn, coordenadas óptimas y el factor de aceleración, dos por la prueba de la media mínima de las correlaciones parciales al cuadrado y tres por la media mínima de las correlaciones parciales elevadas a la cuarta potencia y el criterio de Kaiser.

$\mathrm{Al}$ extraer un factor, se explicó el $47,4 \%$ de la varianza total. El factor tuvo una consistencia interna muy alta $(\alpha$ ordinal $=0,924)$ y validez convergente $(\mathrm{VME}=0,474$ y $\mathrm{CC}=0,925)$ (tabla 2$)$.

$\mathrm{Al}$ extraer dos factores, se explicó el 56,6 \% de la varianza total. El primer factor quedó configurado por 8 indicadores (ítems $2,8,9,10,11,12,13$ y 14), y por su contenido se denominó impacto psicosocial con una consistencia interna muy alta ( $\alpha$ ordinal $=0,918)$, validez convergente $(\mathrm{VME}=0,590$ y $\mathrm{CC}=0,919)$ y discriminante por el criterio de Fornell-Larcker (VME $=0,590>\mathrm{r}^{2}=0,473$ ). El segundo factor quedó constituido por 6 indicadores (ítems 1, 3, 4, 5, 6 y 7), por su contenido se denominó impacto físico y tuvo una consistencia interna alta $(\alpha$ ordinal $=0,825)$ y validez convergente $(\mathrm{VME}=0,455$ y CC $=0,830$ ), pero careció de validez discriminante por el criterio de Fornell-Larcker $\left(\operatorname{VME}=0,455<\mathrm{r}^{2}=0,473\right)$. Por el criterio de las CHRMR hubo validez discriminante entre ambos factores (CHRMR $=0,795<0,85)$ (tabla 2).

$\mathrm{Al}$ extraer tres factores, se explicó el $61,5 \%$ de la varianza total. El primer factor quedó conformado por ocho indicadores (ítems 2, 8, 9, 10,11,12, 13 y 14), por su contenido se denominó impacto psicosocial y tuvo una consistencia interna muy alta ( $\alpha$ ordinal $=0,918)$ y validez convergente $(\mathrm{VME}=0,603$ y CC $=0,919)$. El segundo factor quedó conformado por dos indicadores (ítems 5 y 6 ), por su contenido se denominó sintomas de mal olor y sabor de boca o discapacidad física, siguiendo a Slade (3), y tuvo una consistencia interna alta $(\alpha$ ordinal $=0,879)$ y validez convergente $(\mathrm{VME}=0,771$ y $C C=0,871)$. El tercer factor quedó configurado por cuatro indicadores (ítems 1, 3, 4 y 7); por su contenido de inflamación, sangrado y dolor de encía y dientes sensibles se denominó impacto físico, y su consistencia interna fue aceptable ( $\alpha$ ordinal $=0,788$ ) y presentó validez convergente $(\mathrm{VME}=0,509$ y $\mathrm{CC}=0,803)$. La varianza compartida entre los tres factores varió de 0,469 a 0,291, siendo la VME menor en todos los casos, por lo que tuvieron validez discriminante por el criterio de Fornell-Larcker. También mostraron validez discriminante por el criterio de las CHRMR (tabla 2).

TABLA 2

Matriz de un factor y matrices estructurales de dos y tres factores $\mathrm{n}=256$. Correlaciones policóricas. Extracción: mínimos cuadrados ponderados. Rotación: Promax (generando la hipótesis estructural por Varimax). NI = número de factores destacados en negrilla, $\mathrm{VME}=$ varianza media extraída, $\alpha=$ coeficiente alfa ordinal y CC $=$ confiabilidad compuesta.

\begin{tabular}{lcccccc}
\hline \multirow{2}{*}{ Ítems } & \multirow{2}{*}{ F1 } & \multicolumn{2}{c}{ 2 factores } & \multicolumn{3}{c}{ 3 factores } \\
\cline { 3 - 7 } & & F1 & $\mathbf{F 2}$ & F1 & F2 & F3 \\
\hline i1 & $\mathbf{0 , 6 1 3}$ & 0,483 & $\mathbf{0 , 8 5 6}$ & 0,451 & 0,533 & $\mathbf{0 , 8 2 3}$ \\
i2 & $\mathbf{0 , 7 3 8}$ & $\mathbf{0 , 7 0 9}$ & 0,659 & $\mathbf{0 , 7 0 0}$ & 0,459 & 0,654 \\
i3 & $\mathbf{0 , 6 4 9}$ & 0,578 & $\mathbf{0 , 6 8 7}$ & 0,572 & 0,314 & $\mathbf{0 , 7 8 0}$ \\
i4 & $\mathbf{0 , 5 2 1}$ & 0,473 & $\mathbf{0 , 5 1 5}$ & 0,469 & 0,260 & $\mathbf{0 , 5 6 5}$ \\
i5 & $\mathbf{0 , 6 1 9}$ & 0,546 & $\mathbf{0 , 6 5 0}$ & 0,504 & $\mathbf{0 , 8 6 0}$ & 0,531 \\
i6 & $\mathbf{0 , 6 3 1}$ & 0,567 & $\mathbf{0 , 6 3 2}$ & 0,534 & $\mathbf{0 , 8 9 6}$ & 0,499 \\
i7 & $\mathbf{0 , 5 4 9}$ & 0,457 & $\mathbf{0 , 6 6 1}$ & 0,434 & 0,416 & $\mathbf{0 , 6 5 8}$ \\
i8 & $\mathbf{0 , 6 4 7}$ & $\mathbf{0 , 6 7 5}$ & 0,478 & $\mathbf{0 , 6 7 2}$ & 0,435 & 0,431 \\
i9 & $\mathbf{0 , 8 5 3}$ & $\mathbf{0 , 8 2 9}$ & 0,727 & $\mathbf{0 , 8 1 2}$ & 0,610 & 0,672 \\
i10 & $\mathbf{0 , 7 9 7}$ & $\mathbf{0 , 7 9 0}$ & 0,650 & $\mathbf{0 , 7 7 8}$ & 0,529 & 0,611 \\
i11 & $\mathbf{0 , 7 9 7}$ & $\mathbf{0 , 8 7 0}$ & 0,550 & $\mathbf{0 , 8 8 5}$ & 0,403 & 0,549 \\
i12 & $\mathbf{0 , 7 6 9}$ & $\mathbf{0 , 8 3 6}$ & 0,535 & $\mathbf{0 , 8 4 0}$ & 0,496 & 0,485 \\
i13 & $\mathbf{0 , 7 6 5}$ & $\mathbf{0 , 7 8 3}$ & 0,609 & $\mathbf{0 , 7 9 4}$ & 0,373 & 0,635 \\
i14 & $\mathbf{0 , 5 9 1}$ & $\mathbf{0 , 6 2 0}$ & 0,432 & $\mathbf{0 , 6 2 2}$ & 0,352 & 0,408 \\
\hline NI & 14 & 8 & 6 & 9 & 2 & 4 \\
VME & 0,474 & 0,590 & 0,455 & 0,603 & 0,771 & 0,509 \\
a & 0,924 & 0,918 & 0,825 & 0,918 & 0,879 & 0,788 \\
CC & 0,925 & 0,919 & 0,830 & 0,919 & 0,871 & 0,803 \\
\hline
\end{tabular}




\section{Análisis factorial confirmatorio}

Se contrastaron los modelos de Silveira y colaboradores (7), Zucoloto y colaboradores (8) y Montero y colaboradores (9). Asimismo, se contrastaron los resultados del AFE, los modelos de uno, dos y tres factores correlacionados. El de un factor coincide con el propuesto por Mello dos Santos y colaboradores (10) y Moral y Rodríguez (13).

\section{Modelo de Silveira y colaboradores (7)}

Se especificó un modelo jerarquizado de siete factores subordinados a uno general. Los siete factores de orden inferior fueron: limitación funcional (ítems 1 y 2), dolor físico (ítems 3 y 4), discapacidad física (ítems 5 y 6), malestar psicológico (ítems 7 y 8), discapacidad psicosocial (ítems 9 y 10), discapacidad social (ítems 11 y 12) y minusvalía (ítems 13 y 14). Los factores de discapacidad física, psicológica y social tuvieron una consistencia interna alta ( $\alpha$ ordinal de 0,857 a 0,888 ). No obstante, los factores de limitación funcional, dolor físico y minusvalía tuvieron una consistencia interna baja ( $\alpha$ ordinal de 0,682 a 0,689 ) y el factor de malestar psicológico tuvo una consistencia interna inaceptable $(\alpha$ ordinal $=0,358$ ). Hubo problemas de validez discriminante en 8 de las 21 comparaciones por el análisis de las CHRMR. El modelo presentó una solución inadmisible. Se reespecificó como un modelo de 7 factores correlacionados, pero nuevamente la solución fue inadmisible.

\section{Modelo de Zucoloto y colaboradores (8)}

Se especificó un modelo jerarquizado de tres factores de orden superior correlacionados (impacto físico, psicosocial y social) y siete factores de orden inferior (los anteriormente definidos). Los factores de limitación funcional, dolor físico y discapacidad física se especificaron jerarquizados al factor de impacto físico; los factores de malestar psicológico y discapacidad psicosocial al factor de impacto psicosocial; y los factores de discapacidad social y minusvalía al factor de impacto social. El modelo presentó una solución inadmisible.

Como modelo alternativo se especificó uno de tres factores correlacionados: impacto físico (ítems 1, 2, 3, 4, 5 y 6), impacto psicosocial (ítems 7, 8, 9 y 10) e impacto social (ítems 11, 12, 13 y 14). El factor de impacto físico tuvo una consistencia interna alta $(\alpha$ ordinal $=0,823)$ y mostró una validez convergente $(\mathrm{VME}=0,458$ y $\mathrm{CR}=0,834)$, aunque careció de validez discriminante con respecto a los otros dos factores $\left(\mathrm{VME}=0,458<\mathrm{r}^{2}\right.$ IFYIP $=0,848 \mathrm{y} \mathrm{r}^{2}$ IFyIS $\left.=0,554\right)$. El factor de impacto psicosocial tuvo una consistencia interna alta $(\alpha$ ordinal $=0,823)$ y validez convergente $(\mathrm{VME}=0,531$ y $\mathrm{CR}=0,815)$, aunque careció de validez discriminante con respecto a los otros dos factores (VME $=0,531<\mathrm{r}^{2}{ }_{\text {IPyIF }}=0,848$ y r$^{2}{ }_{\text {IPyIS }}=$ $0,784)$. El factor de impacto social tuvo una consistencia interna alta ( $\alpha$ ordinal $=0,891)$ y mostró validez convergente $(\mathrm{VME}=0,648 \mathrm{y} \mathrm{CR}=0,879)$ y validez discriminante con respecto al factor de impacto físico $\left(\mathrm{r}^{2} \mathrm{ISyIF}=0,554\right)$, pero no con respecto al factor de impacto psicosocial $\left(\mathrm{r}^{2}{ }_{\text {ISyIP }}=0,784\right)$. Al aplicar el análisis de las CHRMR, el factor de impacto físico no mostró validez discriminante con respecto al de factor de impacto psicosocial $(\mathrm{CHRMR}=0,952)$, pero sí con respecto al de impacto social $(\mathrm{CHRMR}=0,742)$. Los factores de impacto psicosocial y social no tuvieron validez discriminante uno respecto del otro (CHRMR $=0,919)$. La solución fue admisible y el ajuste del modelo fue bueno por cuatro índices y adecuado por dos (tabla 3, figura 2). 
TABLA 3

Índices de ajuste y parsimonia de los modelos con soluciones admisibles

Matriz de correlaciones policóricas. Método: mínimos cuadrados libres de escala.

Modelos: 3F_Zuc.: impacto psicosocial (ítems 2, 8, 9, 10, 11, 12, 13

y 14), impacto físico (ítems $1,3,4$ y 7 )y discapacidad física (ítems 5 y 6 ) 1F: un factor con todos los residuos independientes.

1F_M1: un factor con correlación entre los residuos de los ítems 5 y 6.

1F_M2: un factor con correlación entre los residuos de los ítems 5-6 y 1-7.

3F_AFE: impacto psicosocial (ítems 2, 8, 9, 10,11,12,13 y 14), discapacidad física (ítems 5 y 6 ) e impacto físico (ítems 1,3,4 y 7)

\begin{tabular}{lcccccccc}
\hline \multirow{2}{*}{ Índices } & \multicolumn{2}{c}{ Interpretación } & \multicolumn{7}{c}{ Modelos } \\
\cline { 2 - 9 } & Bueno & Adecuado & 3F Zuc. & 1F & 1F M1 & 1F M2 & 2F AFE & 3F AFE \\
\hline \multirow{2}{*}{$\chi^{2}$} & & & 152,590 & 204,807 & 159,739 & 136,401 & 150,603 & 105,357 \\
$g l$ & & & 74 & 77 & 76 & 75 & 76 & 74 \\
$\chi^{2} / g l$ & $\leq 2$ & $\leq 3$ & 2,062 & 2,660 & 2,102 & 1,819 & 1,982 & 1,424 \\
SRMR & $<0,08$ & $<0,10$ & 0,076 & 0,088 & 0,077 & 0,071 & 0,075 & 0,065 \\
GFI & $\geq 0,95$ & $\geq 0,90$ & 0,979 & 0,971 & 0,978 & 0,981 & 0,979 & 0,985 \\
AGFI & $\geq 0,90$ & $\geq 0,85$ & 0,970 & 0,961 & 0,969 & 0,973 & 0,971 & 0,979 \\
NFI & $\geq 0,95$ & $\geq 0,90$ & 0,972 & 0,962 & 0,970 & 0,975 & 0,972 & 0,980 \\
RFI & $\geq 0,95$ & $\geq 0,90$ & 0,965 & 0,955 & 0,964 & 0,969 & 0,966 & 0,976 \\
PR & $\geq 0,75$ & $\geq 0,50$ & 0,813 & 0,846 & 0,835 & 0,824 & 0,835 & 0,813 \\
\hline
\end{tabular}

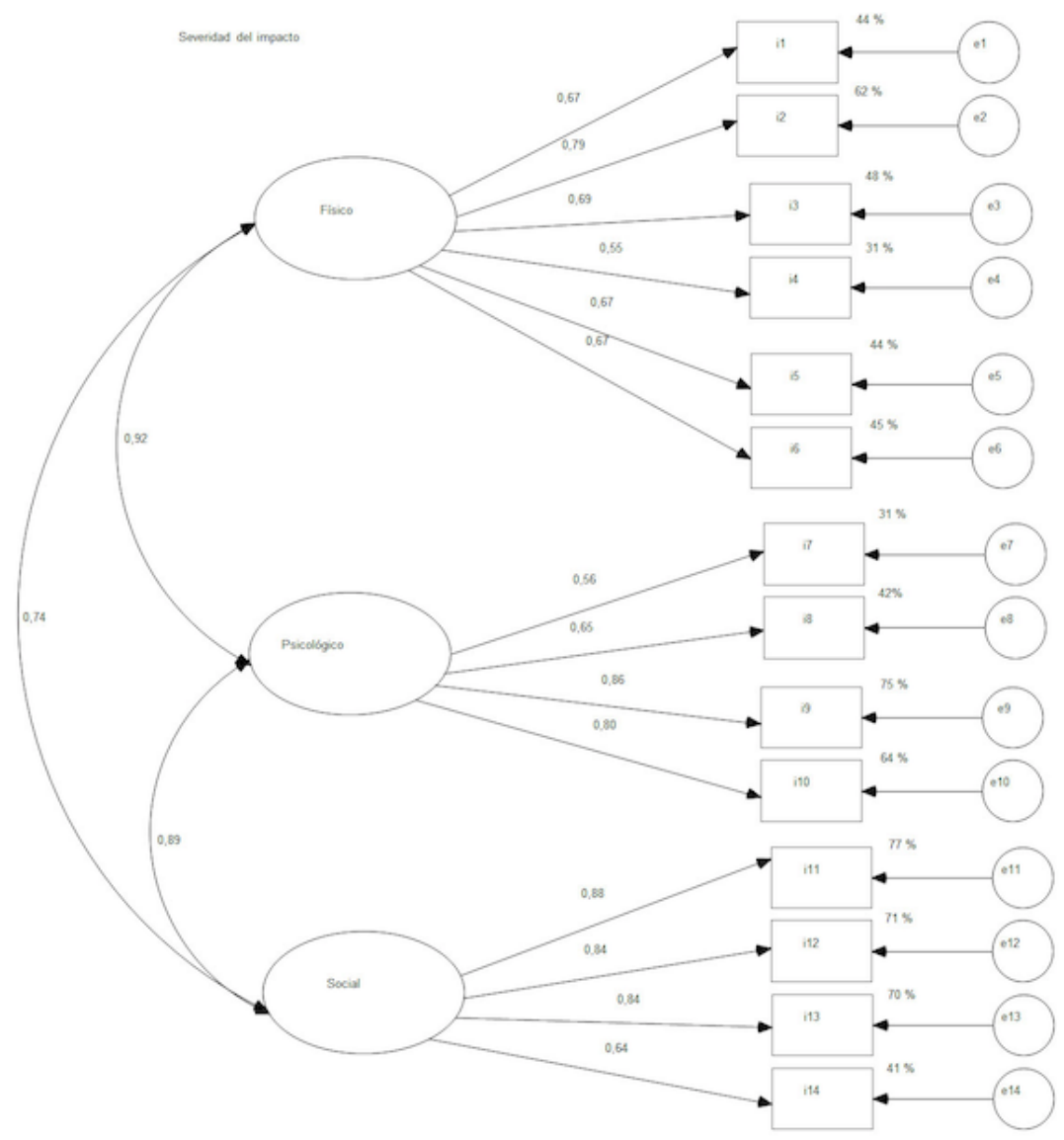

FIGURA 2

Modelo reespecificado de Zucoloto y colaboradores (2014) 


\section{Montero y colaboradores (9)}

Se especificó un modelo de tres factores correlacionados: impacto psicosocial (ítems 5, 6, 9, 10, 11, 12, 13 y 14), dolor/malestar (ítems 3, 4, 7 y 8) y limitación funcional (ítems 1 y 2). La consistencia interna del factor de impacto psicosocial fue muy alta $(\alpha$ ordinal $=0,905)$, pero la de los factores de limitación funcional y malestar/dolor fue baja ( $\alpha$ ordinal $=0,689$ y 0,690, respectivamente). Al aplicar el análisis de las CHRMR, los factores de limitación funcional e impacto psicosocial mostraron validez discriminante $(\mathrm{CHRMR}=$ 0,858), pero el factor de dolor/malestar físico careció de validez discriminante con respecto al de limitación funcional $($ CHRMR $=1,092)$ y al de impacto psicosocial $(C H R M R=0,937)$. El modelo presentó una solución inadmisible. Como alternativa se especificó un modelo de un factor de orden superior y tres factores jerarquizados, pero nuevamente la solución fue inadmisible.

\section{Modelo de un factor $(10,13)$}

La solución fue admisible, el ajuste fue bueno por cuatro y adecuado por dos, la parsimonia fue alta $(\mathrm{PR}=$ $0,846)$ y el factor mostró validez convergente $(\mathrm{VME}=0,474$ y $\mathrm{CC}=0,925)$ (tabla 3 ). Tras revisar la matriz de residuos, se liberó un parámetro, la correlación entre los residuos de los ítems 5 y 6 . Mejoró el ajuste del modelo $\left(\Delta \chi^{2} / \mathrm{gl}=45,062, \Delta \mathrm{NFI}=0,008\right.$ y $\left.\Delta \mathrm{RFI}=0,009\right)$ y este fue bueno por cinco índices y adecuado por uno, se preservó la parsimonia alta $(\mathrm{PR}=0,810)$ y el factor presentó validez convergente $(\mathrm{VME}=0,470$ y $\mathrm{CC}=0,924)$. Al liberar un parámetro adicional, la correlación entre los residuos de los ítems 1 y 7 , mejoró el ajuste en comparación con el modelo con todos los residuos independientes $\left(\Delta \chi^{2} / \mathrm{gl}=68,405\right.$, $\Delta \mathrm{NFI}=0,013$ y $\Delta \mathrm{RFI}=0,014)$. En este modelo, el ajuste fue bueno por los seis índices, se conservó la parsimonia alta $(P R=0,824)$ y el factor tuvo validez convergente $(\mathrm{VME}=0,468$ y $\mathrm{CC}=0,923)$ (tabla 3 , figura 3).

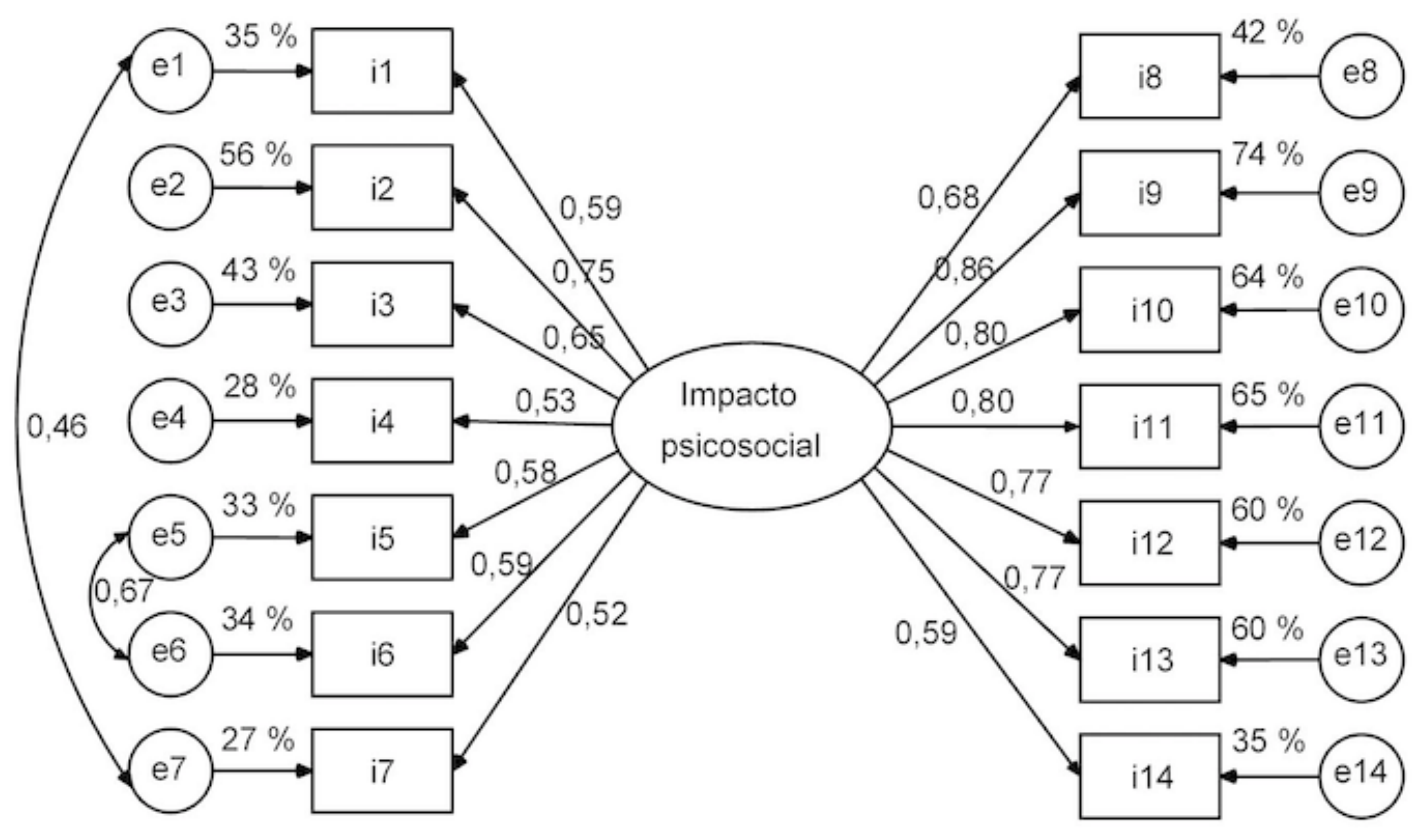

FIGURA 3

Modelo de un factor con dos correlaciones entre residuos de medida 


\section{Modelo de dos factores derivado del AFE}

El factor de impacto psicosocial (ítems 2, 8, 9, 10, 11, 12, 13 y 14) mostró validez convergente (VME = $0,588$ y CR $=0,919)$, pero no discriminante por el criterio de Fornell-Larcker $\left(\mathrm{VME}=0,588<\mathrm{r}^{2}=\right.$ 0,632). El factor de impacto físico (ítems 1, 3, 4, 5, 6 y 7) careció de validez convergente (VME = 0,445 y $\mathrm{CR}=0,827$ ) y discriminante por el criterio de Fornell-Larcker (VME $\left.=0,445<\mathrm{r}^{2}=0,632\right)$. No obstante, hubo validez discriminante entre los dos factores por el análisis de las correlaciones heterorrasgomonorrasgo (CHRMR $=0,795)$. El ajuste fue bueno con base en los 6 índices y la parsimonia alta $(\mathrm{PR}=$ $0,835)$ (tabla 3, figura 4).

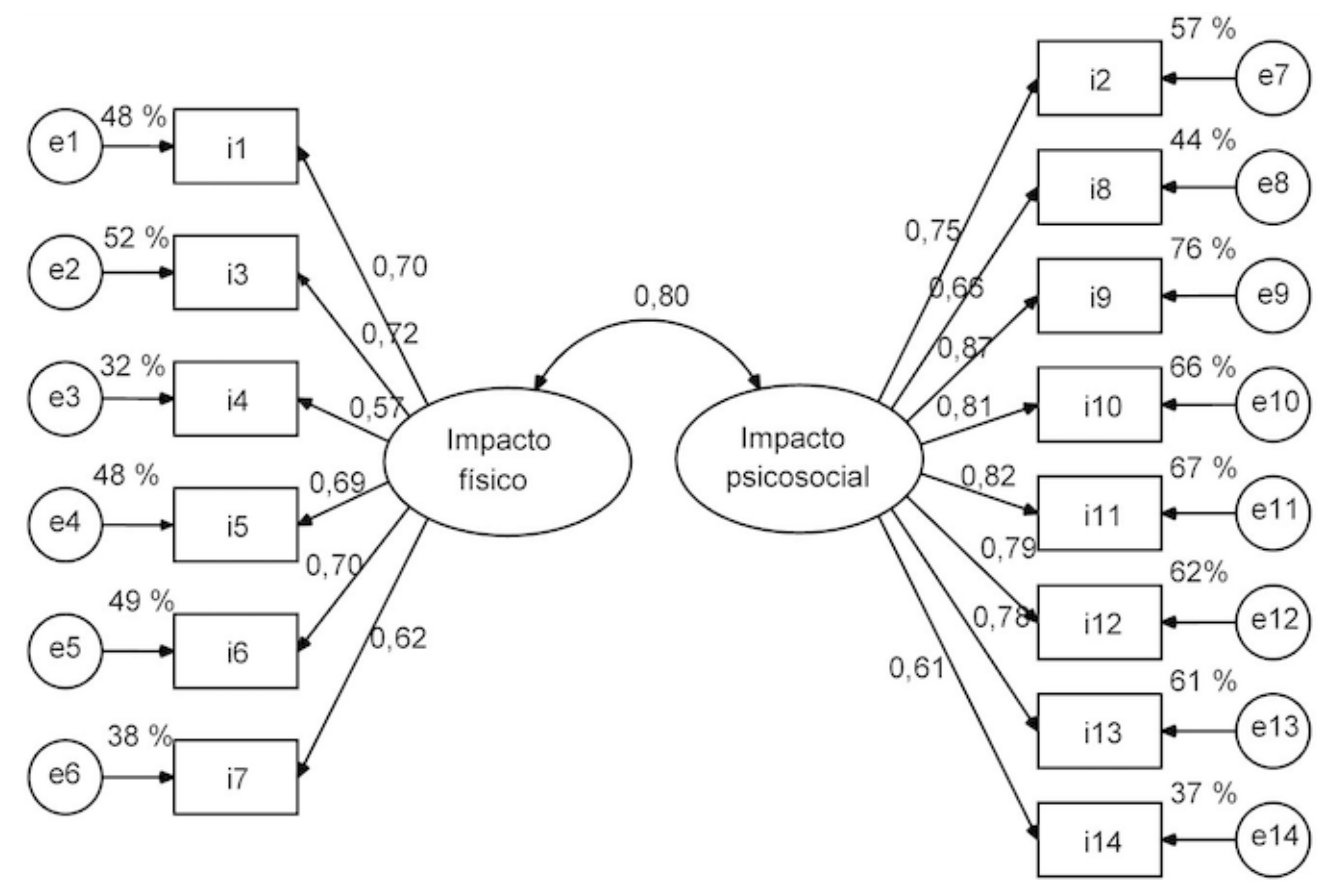

FIGURA 4

Modelo de dos factores correlacionados derivado del AFE

\section{Modelo de tres factores correlacionados derivado del AFE}

El factor de impacto psicosocial (IP: ítems 2, 8, 9, 10, 11, 12, 13 y 14) mostró validez convergente (VME = $0,588$ y CR $=0,919)$ y discriminante con respecto a los otros dos factores (VME $=0,588>r^{2}$ IP y IF $=0,542$ $\mathrm{y} \mathrm{r}^{2}$ DF y IP $\left.=0,396\right)$. El factor de impacto físico (IF: ítems $1,3,4$ y 7 ) careció de validez convergente (VME = $0,492$ y $C R=0,793)$ y validez discriminante en relación con el factor de impacto psicosocial ( $\mathrm{VME}=0,492$ $<\mathrm{r}_{\text {IP y IF }}^{2}=0,542$ ), aunque presentó validez discriminante en cuanto al factor de discapacidad física (VME $=0,492>\mathrm{r}^{2}$ IF y DP $=0,348$ ). El factor de discapacidad física (ítems 5 y 6 ) tuvo validez convergente (VME $=0,784$ y CR $=0,879)$ y discriminante con respecto a los otros dos factores $\left(\mathrm{VME}=0,784>\mathrm{r}^{2}\right.$ DF y IF $=$ 0,348 y r $^{2}$ DF y IP $\left.=0,396\right)$. Al aplicar el análisis de las CHRMR, el factor de impacto físico mostró validez discriminante en relación con la discapacidad física (CHRMR $=0,593)$ y al impacto psicosocial (CHRMR $=0,738)$. Asimismo, los factores de discapacidad física e impacto psicosocial mostraron validez discriminante uno respecto al otro $(\mathrm{CHRMR}=0,627)$. Se ajustó con base en los 6 índices y la parsimonia alta $(\mathrm{PR}=0,813)$ (tabla 3, figura 5). 


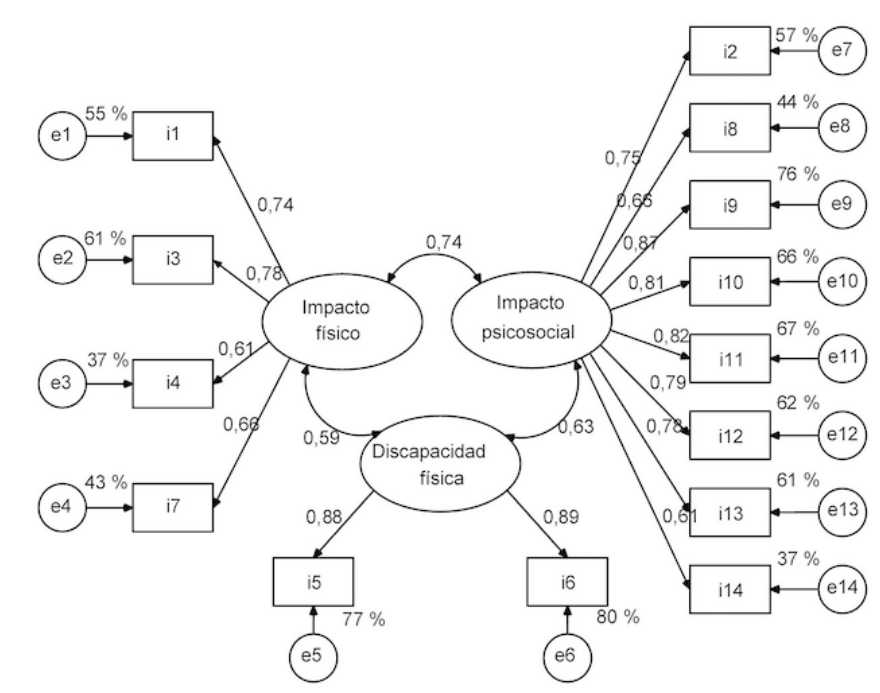

FIGURA 5

Modelo de tres factores correlacionados derivado del AFE

\section{DISCUSIÓN}

Como primer objetivo se formuló estudiar la distribución, la discriminabilidad y la consistencia interna de los ítems y la puntuación total. Los 14 ítems presentaron la propiedad de la discriminabilidad y fueron consistentes, aunque los ítems 4 y 14 tuvieron un valor de homogeneidad por debajo del límite inferior estipulado, esto es, su correlación con el resto de la escala ligeramente menor que 0,50 . No obstante, el hecho de que la consistencia interna de la escala se reduzca con su eliminación y que sus cargas factores sean mayores que 0,50 en el modelo unifactorial indica que sí poseen inconsistencia interna dentro de la escala. Consecuentemente, los 14 ítems se retuvieron.

La tendencia central en los ítems correspondió a una mediana de 1, "casi nuca", y 0, "nunca”. En sus distribuciones dominó la asimetría positiva y en aquellas que presentaron simetría se observó apuntamiento, es decir, las puntuaciones se concentraron en los valores más bajos o en torno a la mediana, que fue muy baja. Los datos reflejan que el impacto de síntomas de enfermedad periodontal es mínimo entre estos adultos de la población general. Además, consecuente con la naturaleza ordinal de los ítems y sus características distribucionales, no hubo normalidad multivariada. El ítem 4 sobre sensibilidad dental fue el que tuvo la mediana más alta ( 2 = "ocasionalmente"), la única asimetría negativa y la homogeneidad más baja; por lo que presentó un comportamiento distinto a los demás ítems, indicando que es relativamente frecuente. Precisamente, es un síntoma no muy específico de enfermedad periodontal y puede corresponder a otras situaciones, como presencia de caries, desgaste del esmalte (atrición, abrasión o erosión dental) por cepillado inadecuado, blanqueamiento dental o ingesta de alimentos ácidos (22).

Con un intervalo de confianza del $95 \%$, la media en la puntuación total del OHIP-14-PD entre estos adultos mexicanos de población general $(12,32[11,29,13,34])$ fue estadísticamente equivalente a la de los adolescentes brasileños de población general $(15,6[10,4,22,8])$ del estudio de Silveira y colaboradores (7) y de los adultos mexicanos que acudían a consulta para tratamiento dental profiláctico (14,54 [12,49, 16,59]) del estudio de Moral y Rodríguez (13). Sin embargo, fue significativamente menor que la de los pacientes mexicanos con periodontitis (20,39 [18,60, 22,18]) del estudio de Moral y Rodríguez (13) y los pacientes españoles con dolor orofacial y trastornos temporomandibulares $(20,57[19,52,21,62])$ del estudio de Blanco y colaboradores (23). Precisamente, el percentil 75 de esta muestra correspondió a la mediana de los pacientes odontológicos mexicanos (con gingivitis o periodontitis) del estudio de Moral y Rodríguez (13). Por consiguiente, una puntuación de 17 se interpreta como la existencia de un efecto en la calidad de vida causado por síntomas de un posible padecimiento periodontal. Debe señalarse que la puntuación total del OHIP-14-PD no siguió una distribución normal, fue asimétrica positiva y apuntada, por lo que debe interpretarse usando percentiles poblacionales. 
El segundo objetivo del presente estudio fue explorar la estructura factorial del OHIP-14-PD. Los análisis revelaron tres posibles modelos. El primero surge de un factor concordante con la propuesta de Mello dos Santos y colaboradores (10) y Moral y Rodríguez (13). Se sustentó con dos criterios sólidos (análisis paralelo de Horn y coordenadas óptimas), aparte del criterio del factor de aceleración, unas cargas factoriales mayores que 0,50 , una consistencia interna mayor que 0,90 y validez convergente.

El segundo es un modelo de dos factores correlacionados sustentado por el criterio de Velicer (correlaciones parciales al cuadrado). En este modelo, el criterio de las CHRMR indicó validez discriminante y ambos factores resultaron consistentes y con validez convergente, pero el factor de impacto físico careció de validez discriminante por el criterio de Fornell-Larcker. Anteriormente, Brennan y Spencer (6) propusieron un modelo de dos factores de limitación funcional-incapacidad física y aspectos psicosociales de la discapacidad y minusvalía. Tales factores difieren en su configuración de los obtenidos en el presente estudio, lo que resultó en que la interpretación de los presentes factores de impacto psicosocial y físico fue más coherente.

Finalmente, se obtuvo un modelo de tres factores correlacionados sustentado por el criterio de Velicer (correlaciones parciales a la cuarta potencia) y la regla de Kaiser que se consideran criterios más débiles (16). Los tres factores tuvieron validez convergente y discriminante. El factor de impacto psicosocial tuvo consistencia interna muy alta y el de discapacidad física tuvo alta. No obstante, la consistencia interna del factor de impacto físico fue aceptable. Como limitación importante cabe señalar que el factor de discapacidad física tuvo solo dos indicadores, que es un número insuficiente para definir el modelo de medida de una variable latente (15). Comparado con el modelo bifactorial, su factor de impacto físico se desdobló en dos factores, uno de dos indicadores (discapacidad física) y el otro con cuatro (impacto físico). Difiere de los tres factores de orden superior propuestos por Zucoloto y colaboradores (8), así como de los tres factores de Montero y colaboradores (9), aunque con este último comparte el tener un factor con solo dos indicadores.

Se definió como tercer objetivo contrastar cuatro modelos con los factores propuestos para el OHIP-14, así como los tres derivados del AFE. Este tercer objetivo se complementó con un cuarto objetivo: comprobar el cumplimiento de las propiedades de consistencia interna y validez convergente y discriminante en los factores de cada modelo. Los modelos jerarquizados de Silveira y colaboradores (7) y Zucoloto y colaboradores (8) y el de tres factores correlacionados de Montero y colaboradores (9) serían descartables por proporcionar soluciones inadmisibles, así como por tener un número insuficiente de indicadores y problemas de consistencia interna, validez convergente y discriminante en algunos de sus factores. El modelo jerarquizado de Zucoloto y colaboradores (8) fue reespecificado en el intento de hallar una solución admisible. Se definió uno de tres factores correlacionados a partir de los tres factores de orden superior que suprimía los siete factores de orden inferior. De este modo, se logró dicho objetivo y un ajuste de bueno a aceptable. No obstante, el problema de falta de validez discriminante entre los factores, evidenciado por el modelo jerarquizado, volvió a estar presente, especialmente entre los factores de impacto psicológico y social. Por tanto, no sería un buen modelo.

El modelo de un factor de Mello dos Santos y colaboradores (10) y Moral y Rodríguez (13) fue sustentado por el AFE, resultó ser el más parsimonioso y presentó una VME ligeramente menor al $50 \%$, aunque su confiabilidad compuesta fue mayor que 0,90 , por lo que se puede considerar que mostró validez convergente (14). La consistencia interna de los 14 ítems fue muy alta y no mejoró con la eliminación de ninguno de ellos, por lo que los ítems 4 y 14 quedaron bien representados dentro de este modelo. Su ajuste varió de bueno a adecuado. El modelo con dos correlaciones entre residuos de medida preservó sus propiedades de consistencia interna muy alta, parsimonia alta y validez convergente, además logró un buen ajuste por los seis índices. Por tanto, sería un buen modelo para el OHIP-14-PD.

La correlación alta entre los residuos de ítems 5 y 6 del modelo unifactorial corregido revela el factor subyacente que emerge en la solución trifactorial, constituido por los ítems sobre mal olor y sabor de la boca. Este factor, denominado discapacidad física, ya aparecía en el modelo inicial de la escala (4), en un estudio realizado con una muestra comunitaria australiana, el cual se retomó y validó posteriormente por parte de 
Silveira y colaboradores (7) y Zucoloto y colaboradores (8), usando una muestra comunitaria de adolescentes brasileños, el primero, y una muestra clínica odontológica de adultos jóvenes brasileños, el segundo. Además, esta corrección también fue utilizada en el estudio de Moral y Rodríguez (13) para obtener un ajuste perfecto del modelo unifactorial en una muestra de pacientes odontológicos. Así, parece que estos síntomas son percibidos como un problema específico y distintivo por los respondientes en distintos países y poblaciones, por lo que se requiere destacarlos de forma específica dentro del modelo de un factor. Debe señalarse que se entiende por discapacidad toda restricción de la capacidad de realizar una actividad (física, de relación o comunicación social) en la forma o dentro del margen considerado normal, debido a los síntomas de la enfermedad $(4,5)$.

La correlación moderada entre los ítems 1 y 7 sobre percepción de encía inflamada con mal aspecto que dificulta el cepillado a causa del sangrado no se ha destacado en estudios anteriores. Así, parecen ser síntomas percibidos como un problema específico y distintivo por los respondientes mexicanos y que requieren diferenciarse dentro del modelo de un factor general.

Al estar configurados por sólo dos ítems, resulta más adecuado contemplar a estos dos potenciales factores como dos correlaciones entre los residuos, esto es, como aspectos no explicados por el factor general de severidad del impacto de la enfermedad periodontal, que especificarlos como factores aislados. Precisamente, el factor de limitación funcional, que destacó en el estudio de Montero y colaboradores (9), en el presente estudió tuvo una consistencia interna baja y no se configuró de forma aislada, mostrando que contemplar factores tan limitados en indicadores es difícil de replicar y puede generar soluciones inadmisibles en otras poblaciones.

El modelo de dos factores correlacionados derivado del AFE tuvo un número suficiente de indicadores en cada factor, un buen ajuste, una parsimonia alta, incluso mayor que la del modelo de un factor con dos correlaciones entre sus residuos de medida, presentó validez discriminante por el criterio de las CHRMR y el factor de impacto psicosocial tuvo validez convergente. No obstante, presentó debilidades. El factor de impacto físico careció de validez convergente y ambos factores no tuvieron validez discriminante por el criterio de Fornell-Larcker.

El modelo de tres factores presentó el mejor ajuste, parsimonia alta, validez discriminante por el criterio de las CHRMR, así como validez convergente y discriminante por el criterio de Fornell-Larcker en los factores de impacto psicosocial y discapacidad física. A pesar de estas propiedades tan favorables, su mayor debilidad reside en el hecho de contar con un número insuficiente de indicadores en el factor de incapacidad física como previamente se señaló. Además, el factor de impacto físico careció de validez convergente y no tuvo validez discriminante con respecto al de impacto físico por el criterio de Fornell-Larcker.

Como limitación del presente estudio se tiene el uso de un muestreo no probabilístico, por lo que los resultados no se pueden generalizar como estimaciones paramétricas, sino que deben manejarse como hipótesis en futuros estudios en población general de una ciudad industrial del norte de México. La utilidad de los resultados obtenidos recaba en dilucidar la estructura factorial y consistencia interna de los factores del OHIP-14 en su aplicación a la enfermedad periodontal. Como fortalezas se tiene el uso de una muestra con un tamaño adecuado (al menos 7 participantes por parámetro que se vaya a estimar y con más de 200 participantes) y técnicas de análisis adecuadas a la naturaleza ordinal de las variables.

\section{CONCLUSIÓN}

Los 14 ítems presentaron discriminabilidad y se pueden considerar consistentes, aún los ítems 4 y 14, que fueron los más débiles en esta última propiedad. La consistencia interna de los 14 ítems fue muy alta. La distribución de la escala presentó asimetría positiva y apuntamiento, al no seguir una distribución normal. La tendencia central y la distribución de los ítems y la escala refleja un impacto mínimo de síntomas de 
periodontitis en la salud bucal, conforme con una naturaleza no clínica de la muestra. Finalmente, el modelo de un factor no solo presentó el apoyo más fuerte por los criterios para determinar el número de factores en el AFE, sino que su ajuste fue de bueno a aceptable en el AFC, tuvo validez convergente y la parsimonia más alta.

\section{RECOMENDACIONES}

Se recomienda manejar el OHIP-14-PD como una medida unidimensional. Se sugiere reproducir la investigación usando una muestra probabilística. Se propone usar el modelo de un factor con una corrección (correlación entre los residuos de los ítems 5 y 6) como el mejor modelo, desarrollar normas de interpretación de la escala usando puntuaciones de percentil, validar la puntuación de 17 como punto de corte para detectar casos de impacto de la enfermedad periodontal sobre la salud bucal, así como estudiar la validez concurrente en relación con hábitos de higiene bucal y variables sociodemográficas.

\section{AGRADECIMIENTOS}

Este estudio fue motivado por la necesidad de validar el OHIP-14, para su aplicación en una investigación de tesis doctoral sobre el efecto de la depresión como factor de riesgo de periodontitis crónica en adultos mexicanos. Recibió apoyo de las facultades de Odontología y Psicología de la Universidad Autónoma de Nuevo León. La segunda autora contaba con una beca para estudios de doctorado concedida por el Sistema Nacional de Ciencia y Tecnología (Conacyt) de México.

Agradecimientos a Elsa Adriana Pacheco Juárez, Irma Aurora Rodríguez Franco, Víctor Hugo Dávila Villarreal, Ricardo Rodríguez Franco y Lilia Solís Rodríguez, quienes participaron en la recolección de datos.

\section{REFERENCIAS}

1. Sistema de Vigilancia Epidemiológica de Patologías Bucales (SIVEPAB). 10 años vigilando la salud bucal de los mexicanos [internet]. Ciudad de México, México: Sivepab; 2015 [citado 22 diciembre 2017]. Disponible en: h ttp://www.cenaprece.salud.gob.mx/programas/interior/saludbucal/descargas/pdf/10Anos_SIVEPAB.pdf

2. Slade GD, Spencer AJ. Development and evaluation of the Oral Health Impact Profile. Community Dent Health. 1994 Mar; 11(1): 3-11.

3. Slade GD. Derivation and validation of a short-form oral health impact profile. Community Dent Oral Epidemiol. 1997 Aug; 25(4): 284-92.

4. World Health Organization (WHO). ICIDH: International classification of impairments, disabilities, and handicaps [internet]. Geneva, Switzerland: WHO; 1980 [citado 22 diciembre 2017]. Disponible en: http://ap ps.who.int/iris/bitstream/10665/41003/1/9241541261_eng.pdf

5. Locker D. Measuring oral health: a conceptual framework. Community Dent Health. 1988 Mar; 5(1): 3-18.

6. Brennan DS, Spencer AJ. Dimensions of oral health related quality of life measured by EQ-5D+ and OHIP-14. Health Qual Life Outcomes [internet]. 2004 Jul [citado 22 diciembre 2017]; 2: 35. Disponible en: http://hqlo .biomedcentral.com/articles/10.1186/1477-7525-2-35

7. Silveira MF, Marôco JP, Freire RS, Martins AM, Marcopito LF. Impact of oral health on physical and psychosocial dimensions: an analysis using structural equation modeling. Cad Saúde Pública. 2014 Jun; 30(6): 1169-82.

8. Zucoloto ML, Maroco J, Campos JADB. Psychometric properties of the Oral Health Impact Profile and new methodological approach. J Dent Res. 2014 Jul; 93(7): 645-50.

9. Montero J, Bravo M, Vicente MP, Galindo MP, López JF, Albaladejo A. Dimensional structure of the oral healthrelated quality of life in healthy Spanish workers. Health Qual Life Outcomes [internet]. 2010 Feb [citado 22 diciembre 2017] ; 8: 24.. Disponible en: https://www.ncbi.nlm.nih.gov/pmc/articles/PMC2833145/ 
10. Mello dos Santos C, de Oliveira BH, Nadanovsky P, Balbinot Hilgert J, Keller Celeste R, Hugo FN. The Oral Health Impact Profile14: a unidimensional scale? Cad Saúde Pública. 2013 Apr; 29(4): 749-57.

11. Gabardo MCL, Moysés ST, Moysés S. Autopercepção de saúde bucal conforme o Perfil de Impacto da Saúde Bucal (OHIP) e fatores associados: revisão sistemática. Rev Panam Salud Pública. 2013 Jun; 33(6): 439-45.

12. Rodríguez NI, Moral J. Adaptation and content validity by expert judgment of the Oral Health Impact Profile applied to Periodontal Disease. J Oral Res. 2017 Apr; 6(4): 92-6.

13. Moral J, Rodríguez NI. Validation of the Oral Health Impact Profile applied to periodontal patients. Rev Fac Odontol Univ Antioq. 2017 Dic; 29(1): 148-72.

14. Garson GD. Validity and reliability. Asheboro, NC, USA: Statistical Associates Publishers; 2016.

15. Lloret $S$, Ferreres A, Hernández A, Tomás I. El análisis factorial exploratorio de los ítems: una guía práctica, revisada y actualizada. An Psicol. 2014 Oct; 30(3): 1151-69.

16. Courtney MGR. Determining the number of factors to retain in EFA: using the SPSS R-Menu v2.0 to make more judicious estimations. Practical Assess Res Eval. 2013 Apr; 18(8): 1-13.

17. Byrne BM. Structural equation modeling with Amos: Basic concepts, applications, and programming. 3rd ed. New York, NY, USA: Routledge; 2016.

18. Ellis P. The essential guide to effect sizes. New York, NY, USA: Cambridge University Press; 2010.

19. Preacher KJ. Quantifying parsimony in structural equation modeling. Multivariate Behav Res. 2006 Sep; 41(3): 227-59.

20. Farrell AM. Insufficient discriminant validity: a comment on Bove, Pervan, Beatty, and Shiu (2009). J Bus Res. 2010 Mar; 63(3): 324-7.

21. Henseler J, Ringle CM, Sarstedt M. A new criterion for assessing discriminant validity in variance-based structural equation modeling. J Acad Marketing Sci. 2015 Aug; 43(1), 115-35.

22. Taha $\mathrm{S}$, Clarkson BH. Clinician's guide to the diagnosis and management of tooth sensitivity. Berlin, Germany: Springer Verlag; 2014.

23. Blanco A, Blanco A, Biedma L, Serrano R, González L, Blanco E, Segura R. Application of an oral health-related quality of life questionnaire in primary care patients with orofacial pain and temporomandibular disorders. Med Oral Patol Oral Cir Bucal. 2014 Mar; 19(2): e127-e135.

\section{Licencia Creative Commons CC BY 4.0}

Cómo citar: Moral de la Rubia J, Rodríguez Franco NI. Consistencia interna y estructura factorial del Perfil de Impacto de Salud Oral aplicado a la enfermedad periodontal en una muestra de población general de adultos mexicanos. Univ Odontol. 2017 jul-dic; 36(77). DOI: https://doi.org/10.11144/Javeriana.uo36 -77.cief 